NOUVELLE

\title{
Dissémination locale du cancer de la prostate
}

Un chemin pavé de gras

Victor Laurent ${ }^{1}$, Adrien Guérard ${ }^{1}$, Aurélie Toulet ${ }^{1}$, Philippe Valet ${ }^{2}$, Bernard Malavaud $^{3}$, Catherine Muller ${ }^{1}$

Le pronostic du cancer de la prostate est aggravé par l'obésité

Malgré les progrès réalisés en termes de dépistage, de diagnostic et de traitement, le cancer de la prostate demeure, chez l'homme, le cancer le plus fréquent et occupe la deuxième place en termes de mortalité dans les pays développés. L'identification des facteurs de risque de développement de cette maladie, ainsi que des mécanismes impliqués, est un enjeu majeur dans la prise en charge de cette pathologie. La prévalence de l'obésité a plus que doublé au niveau mondial ces 30 dernières années et, si l'obésité n'augmente pas l'incidence du cancer de la prostate, elle est associée à l'apparition de cancers agressifs présentant une augmentation de la dissémination locale et à distance [1]. Comment expliquer ce lien? Dans un contexte d'obésité, l'accumulation de tissu graisseux, en particulier au niveau viscéral, s'accompagne d'une modification du taux circulant de nombreuses molécules biologiquement actives sécrétées par le tissu adipeux (TA). Ces molécules, appelées adipokines et dont font partie la leptine, l'adiponectine et certaines molécules pro-inflammatoires comme I'interleukine 6 (IL6) [2], sont susceptibles d'affecter le comportement des tumeurs [3] $(\rightarrow)$.

Dans le cancer de

la prostate, aucune

étude clinique

$\rightarrow$ Voir la Synthèse de V. Laurent et al., $m / s n^{\circ} 4$, avril 2014 , page 398

robuste n'a associé les taux circulants de ces adipokines à l'apparition de cancers agressifs. Le mécanisme impliqué pourrait donc être de nature paracrine plus qu'endocrine. Ainsi, nos travaux associés à ceux d'autres équipes ont montré, en particulier dans le cancer du sein, le rôle déterminant que joue le tissu adipeux à proximité des tumeurs dans la progression tumorale (pour

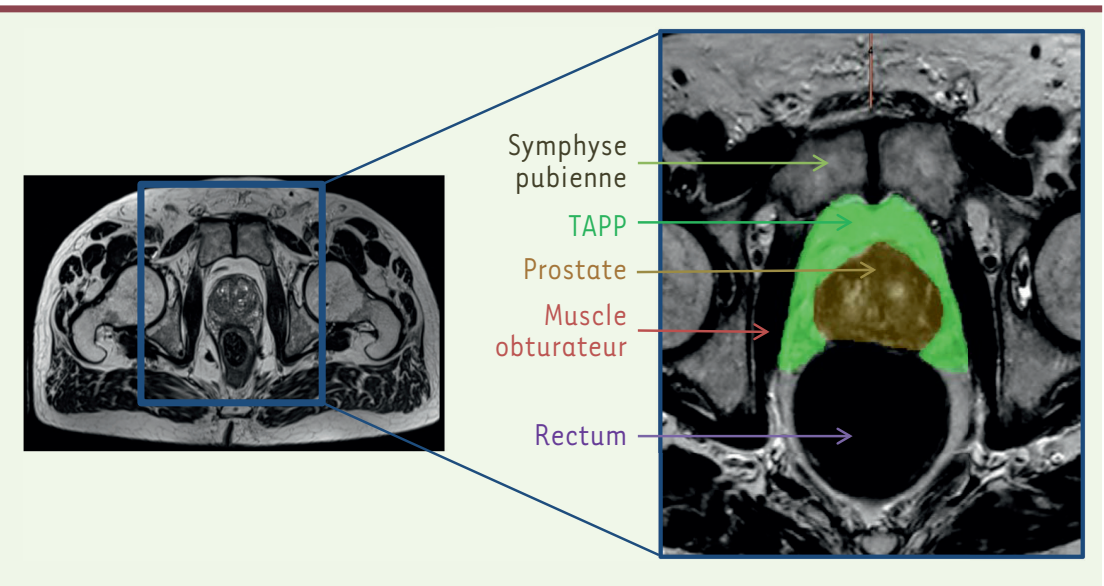

${ }^{1}$ Institut de pharmacologie et de biologie structurale (IPBS), université de Toulouse, CNRS, UPS, 205, route de Narbonne, 31077 Toulouse, France;

${ }^{2}$ Institut des maladies métaboliques et cardiovasculaires (I2MC),

université de Toulouse, Inserm, UPS, 1 , avenue Jean Poulhès, 31077 Toulouse, France ;

${ }^{3}$ Département d'urologie, institut universitaire du cancer, avenue Irène Joliot-Curie, 31059 Toulouse cedex 9, France. muller@ipbs.fr

revue voir [3]). Si la proximité entre la tumeur et le TA est évidente dans le sein, qu'en est-il pour la prostate?

\section{La prostate: une glande}

dans un «îlot » graisseux

La prostate est entourée d'un dépôt graisseux appelé tissu adipeux périprostatique (TAPP), que l'on peut aisément visualiser par imagerie (Figure 1). Ce tissu adipeux est classé comme un tissu adipeux viscéral (TAV) bien que très peu d'études aient réellement caractérisé ses capacités fonctionnelles et sécrétoires. Cliniquement, I'infiltration du TAPP par les cellules cancéreuses est considérée comme un facteur de mauvais pronostic [4] et des études cliniques émergentes suggèrent qu'il pourrait exister un lien entre l'abondance du TAPP et l'agressivité du cancer

Figure 1. La prostate est entourée d'un dépôt graisseux : le tissu adipeux périprostatique. L'étude de l'anatomie de la prostate et des tissus environnants peut se faire par imagerie par résonance magnétique sur des coupes axiales. Sur cette coupe, on peut identifier un organe central : la prostate (zone jaune). Cet organe est entouré d'autres tissus, composés majoritairement par un dépôt adipeux, appelé tissu adipeux périprostatique (TAPP) (zone verte). Ce TAPP est limité par la symphyse pubienne en avant, le rectum en arrière et les muscles obturateurs latéralement. La prostate et le TAPP sont facilement identifiables en imagerie, grâce à leurs coefficients d'atténuation différents qui permettent d'obtenir un signal propre à chacun de ces deux tissus. 


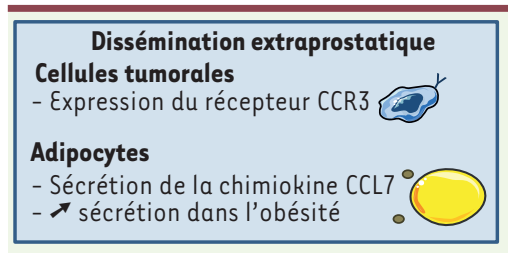

Adipocytes périprostatiques chez le sujet normo-pondéral

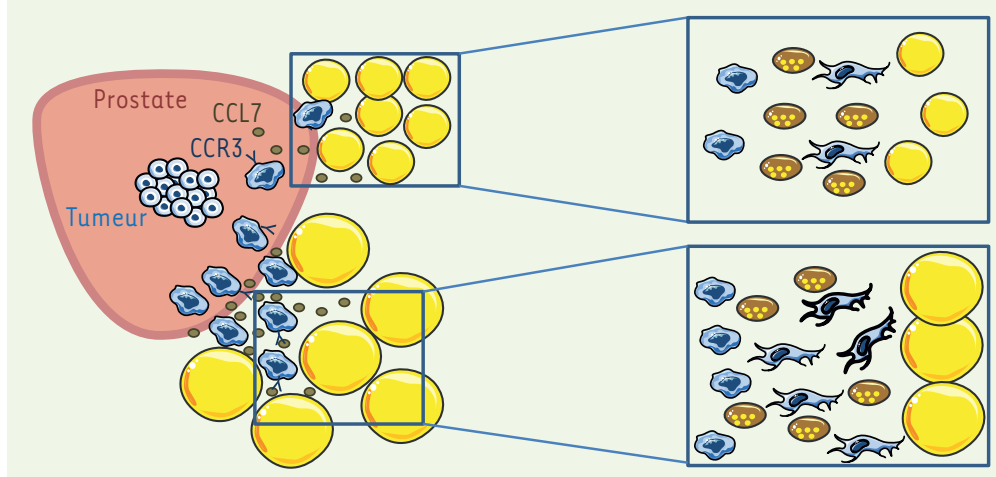

Adipocytes périprostatiques chez le sujet obèse
Dialogue bidirectionnel Cellules tumorales - capacités invasives

Adipocytes

- Délipidation

Phénotype pro-inflammatoire $80^{\circ}$

Figure 2. Rôle paracrine des adipocytes périprostatiques dans la dissémination locale du cancer de la prostate. Les adipocytes du TAPP sécrètent la chimiokine CCL7 qui va diffuser au travers de la capsule prostatique jusqu'à la tumeur. L'interaction de CCL7 avec son récepteur CCR3 exprimé à la surface des cellules tumorales va permettre la migration de ces dernières en dehors de la glande prostatique. Les adipocytes hypertrophiques sécrètent en plus grande quantité la chimiokine CCL7, aboutissant à une augmentation du chimiotactisme dépendant des adipocytes. Ce mécanisme permet d'expliquer pourquoi la dissémination extraprostatique est plus élevée chez le sujet obèse. Dans un second temps, après l'infiltration du TAPP par les cellules tumorales, un dialogue bidirectionnel entre les deux populations va se mettre en place. Sous l'influence des cellules tumorales,

le phénotype des adipocytes est profondément remanié. Ces derniers vont notamment présenter une délipidation importante et une sécrétion accrue de molécules pro-inflammatoires. Ces modifications vont avoir pour conséquence d'augmenter en retour les capacités invasives des cellules cancéreuses. CCL7 : chemokine (C-C motif) ligand 7 ; CCR3 : chemokine ( $C-C$ motif) receptor 3 ; TAPP : tissu adipeux périprostatique.

de la prostate [5]. Ces études, bien que préliminaires, renforcent l'idée d'un rôle paracrine du TAPP dans la progression du cancer de la prostate, rôle qui pourrait être amplifié dans un contexte d'obésité. Les sécrétions du TAPP semblent avoir un effet sur le comportement des cellules prostatiques en stimulant leur prolifération ainsi que leurs capacités migratoires et invasives et cet effet est régulé par l'obésité [6]. De façon très intéressante, cette influence est moins prononcée pour les sécrétions du TAV que pour celles du TAPP suggérant, pour ce dernier, un profil de sécrétion spécifique. Parmi les acteurs impliqués dans cet effet, on retrouve principalement I'IL6 et les métalloprotéases ${ }^{1} 2$ et 9 [6]. Les sécrétions du TAPP pourraient donc influencer les propriétés invasives des cellules tumorales bien que ce concept n'ait pas été validé in vivo. Dans ce contexte, nous avons émis une hypothèse originale sur

${ }^{1}$ Famille d'enzymes de la classe des peptidases dont le nom vient de la présence d'un ion métallique dans leur site actif. le rôle du TAPP. En effet, il est connu que le TA, et en particulier les adipocytes, sécrètent des chimiokines, petites molécules capables de stimuler la migration dirigée de nombreuses cellules [2]. La sécrétion de certaines chimiokines est augmentée dans l'obésité et participe à l'état sub-inflammatoire du TA en permettant le recrutement de cellules du système immunitaire [7]. Les récepteurs à chimiokines étant exprimés par les cellules cancéreuses [8], ce mécanisme pourrait expliquer comment le TAPP favorise une étape clé dans le cancer de la prostate, le franchissement de la capsule prostatique et donc l'envahissement local de la tumeur, l'obésité favorisant ce processus.

\section{L'axe CCR3/CCL7 est déterminant pour} la dissémination locale du cancer de la prostate dans un contexte d'obésité Dans un premier temps, nous avons montré qu'in vitro, les sécrétions adipocytaires sont capables de favoriser la migration dirigée de lignées de cancer de la prostate et que cet effet est amplifié par l'obésité. Cette migration dirigée dépend de l'axe CCR3 (chemokine $[\mathrm{C}-\mathrm{C}$ motif] receptor 3$)$, exprimé par les cellules prostatiques, et son ligand CCL7/MCP3 (chemokine [C-C motif] ligand 7 , aussi appelé monocyte-specific chemokine 3) sécrété par les adipocytes. In vitro, l'effet de l'obésité est totalement aboli par des anticorps bloquants dirigés contre ces deux protéines. Ces résultats ont été confirmés in vivo. En effet, l'injection en intra-prostatique de cellules tumorales n'exprimant plus CCR3 inhibe très fortement la progression tumorale, surtout chez les souris obèses, préservant le TAPP d'une infiltration tumorale. Enfin, chez les patients, l'expression de CCR3 (régulée par l'obésité) est corrélée à l'apparition de tumeurs agressives présentant une extension locale, et est associée à une résistance au traitement chirurgical et à une rechute biologique plus précoce [9]. Ce travail confirme ainsi que l'infiltration des cellules cancéreuses prostatiques dans le TAPP environnant est sous le contrôle 
des sécrétions de ce dernier et que cet effet est amplifié dans l'obésité.

\section{Conclusions et perspectives}

Ce travail met en exergue un nouveau rôle du tissu adipeux dans la progression tumorale. Ce mécanisme, impliquant la migration dirigée dans l'envahissement local, pourrait être étendu à d'autres tumeurs qui présentent un TA de proximité, comme par exemple le cancer du sein, du côlon, du pancréas ou le mélanome. Il est intéressant de signaler que l'axe CCR3/CCL7 semble spécifique du cancer de la prostate [9] et que, si les adipocytes sont effectivement capables d'attirer de très nombreux types de cellules tumorales, les couples récepteurs à chimiokines/ chimiokines impliqués restent à identifier et pourraient être différents pour chaque type de tumeur. Dans le cancer de la prostate, l'inhibition de CCR3 pourrait présenter un intérêt thérapeutique. En effet, bloquer l'infiltration du TAPP est d'autant plus important qu'une fois les cellules cancéreuses présentes dans le TA, un dialogue bidirectionnel s'instaure entre celles-ci et les adipocytes, favorisant ainsi l'invasion tumorale (et donc le processus métastatique) via la capacité des adipocytes à (1) sécréter des molécules pro-inflammatoires, (2) sécréter des protéines de la matrice extracellulaire, (3) remodeler la matrice extracellulaire, mais également (4) modifier le métabolisme des cellules tumorales (pour revue voir [3]) (Figure 2). Notre étude est une des premières à montrer le rôle de CCR3 dans le cancer. Jusqu'alors, ce récepteur, fortement exprimé par les polynucléaires éosinophiles et basophiles, a surtout été impliqué dans des maladies inflammatoires allergiques telles que l'asthme [10]. Des inhibiteurs de CCR3 étant en cours de développement dans cette indication, leur efficacité dans le cancer de la prostate, en particulier chez le sujet obèse, serait intéressante à évaluer. $\diamond$

Dissemination of prostate cancer: a way paved of fat

\section{REMERCIEMENTS}

Les travaux réalisés dans nos équipes ont bénéficié du soutien financier de l'INCA (INCA PL 2010-214, PV et $(M)$, de la Ligue Régionale Midi-Pyrénées Contre le Cancer (CM), de la Fondation de France (PV et CM) et de l'Association pour la Recherche sur les Tumeurs Prostatiques ARTP (CM). Victor Laurent a bénéficié d'une bourse de thèse de l'ARC.
LIENS D'INTÉRÊT

Les auteurs déclarent n'avoir aucun lien d'intérêt concernant les données publiées dans cet article.

\section{RÉFÉRENCES}

1. Parker AS, Thiel DD, Bergstralh $\varepsilon$, et al. Obese men have more advanced and more aggressive prostate cancer at time of surgery than non-obese men after adjusting for screening PSA level and age: results from two independent nested case-control studies. Prostate Cancer Prostatic Dis 2013 ; 16 : 352-6.

2. Ouchi N, Parker JL, Lugus JJ, Walsh K. Adipokines in inflammation and metabolic disease. Nat Rev Immunol 2011; 11 : 85-97.

3. Laurent V, Nieto L, Valet P, Muller C. Tissu adipeux et cancer. Med Sci (Paris) 2014 ; $30: 398-404$.

4. Kapoor J, Namdarian B, Pedersen J, et al. Extraprostatic extension into periprostatic fat is a more important determinant of prostate cancer recurrence than an invasive phenotype.J Urol 2013 ; $190: 2061-6$

5. van Roermund JG, Hinnen KA, Tolman CJ, et al. Periprostatic fat correlates with tumour aggressiveness in prostate cancer patients. BJU Int $2011 ; 107: 1775-9$.

6. Ribeiro R, Monteiro C, Cunha V, et al. Human periprostatic adipose tissue promotes prostate cancer aggressiveness in vitro. J Exp Clin Cancer Res $2012 ; 31: 32$.

7. Xu L, Kitade H, Ni Y, Ota T. Roles of chemokines and chemokine receptors in obesity-associated insulin resistance and nonalcoholic fatty liver disease. Biomolecules 2015; 5 : 1563-79.

8. Balkwill F. Cancer and the chemokine network. Nat Rev Cancer $2004 ; 4: 540-50$.

9. Laurent V, Guérard A, Mazerolles C, et al. Periprostatic adipocytes act as a driving force for prostate cancer progression in obesity. Nat Commun 2016; $7: 10230$.

10. Garcia G, Godot V, Humbert M. New chemokine targets for asthma therapy. Curr Allergy Asthma Rep $2005 ; 5$ : 155-60.

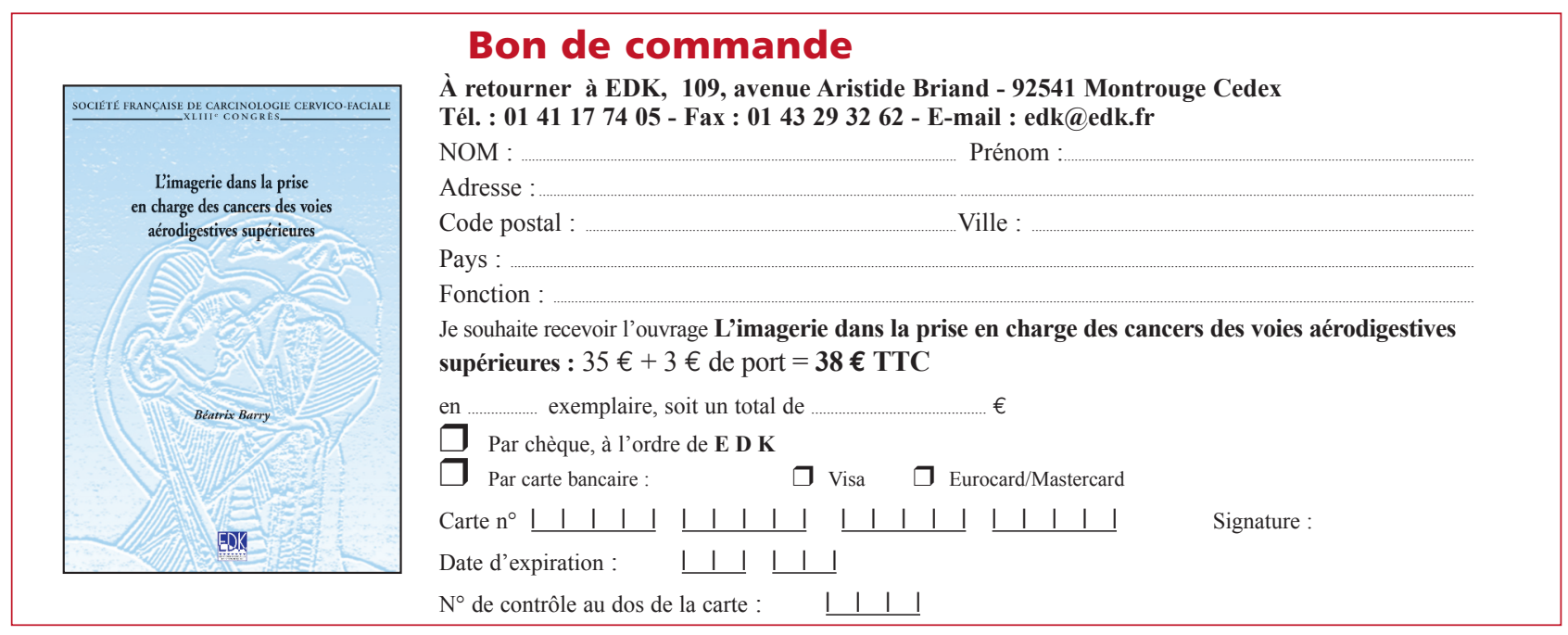

